\title{
Integration of Microdialysis Sampling and Microchip Electrophoresis with Electrochemical Detection
}

\author{
Laura C. Mecker and R. Scott Martin ${ }^{*}$ \\ Saint Louis University, Department of Chemistry, 3501 Laclede Avenue, St. Louis, MO 63103
}

\begin{abstract}
Here we describe the fabrication, optimization, and application of a microfluidic device that integrates microdialysis (MD) sampling, microchip electrophoresis (ME), and electrochemical detection (EC). The manner in which the chip is produced is reproducible and enables the fixed alignment of the MD/ME and ME/EC interfaces. Poly(dimethylsiloxane) (PDMS) -based valves were used for the discrete injection of sample from the hydrodynamic MD dialysate stream into a separation channel for analysis with ME. To enable the integration of ME with EC detection, a palladium decoupler was used to isolate the high voltages associated with electrophoresis from micron-sized carbon ink detection electrodes. Optimization of the ME/EC interface was needed to allow the use of biologically appropriate perfusate buffers containing high salt content. This optimization included changes in the fabrication procedure, increases in the decoupler surface area, and a programmed voltage shutoff. The ability of the MD/ME/EC system to sample a biological system was demonstrated by using a linear probe to monitor the stimulated release of dopamine from a confluent layer of PC 12 cells. To our knowledge, this is the first report of a microchip-based system that couples microdialysis sampling with microchip electrophoresis and electrochemical detection.
\end{abstract}

\section{Introduction}

Microdialysis (MD) sampling is a powerful method for monitoring dynamically changing systems making it especially useful in neurochemistry and pharmacokinetics. ${ }^{1,2}$ In most cases, the analysis of MD samples is performed off-line, meaning the sample is periodically collected in vials and later analyzed. ${ }^{3-6}$ However, the direct on-line coupling of MD to fast analysis techniques like capillary electrophoresis (CE) can enable near real-time monitoring of biological environments. ${ }^{7}{ }^{8}$ Due to the continual collection of sample with on-line analyses, little information is lost, as opposed to off-line techniques where the time needed to collect an aliquot of sample can lead to the loss of dynamic information. To perform on-line analysis with MD sampling, an analytical system that can accommodate small sample volumes is required, as typical flow rates for MD sampling are $1 \mu \mathrm{L} / \mathrm{min}$ or less. A significant amount of work from both Kennedy and Lunte's labs have shown the advantages of coupling traditional CE with MD. ${ }^{7}, 9-11$ However, coupling MD sampling with conventional CE has not been commercialized or widely used in the neurosciences, primarily because the fluidic interface used to couple the two techniques is complex and requires precise manual alignment.

Another issue that arises from the on-line coupling of MD with $\mathrm{CE}$ is the method of detection. Most of the previous studies have utilized fluorescence detection after derivatization of the analytes through a primary amine moiety. ${ }^{7,9,10,12}$ The use of electrochemical detection is an attractive alternative to fluorescence detection in these systems because it can be used to directly detect small molecule neurotransmitters such as catecholamines without 
derivatization. ${ }^{5,13,14}$ However, the coupling of MD with traditional CE and electrochemical (EC) detection is further complicated by the need for two decoupling schemes, one to isolate the MD system from CE and the other to isolate the high voltages of CE from the EC detector. The only example of the on-line coupling of these three components has been performed by Lunte's group at the University of Kansas and involved the use of two hand-made fracture decouplers. ${ }^{11}$

A possible solution to the issues encountered in coupling MD sampling to $\mathrm{CE}$ with $\mathrm{EC}$ detection is the use of microchip-based systems. The use of microfabrication techniques offers a means to fix the alignment and integration of multiple components as well as the ability to mass produce a complex interface. There are several advantages of microchip-based systems, many of which are also beneficial when performing in vitro or in vivo analysis. These include fast analysis times (on the order of seconds), the ability to perform electrophoretic separations, the possibility for portability and disposability, small channel volumes (on the order of nanoliters), and the ability to inject sample volumes as small as picoliters. In order to make an on-line microchip system possible, however, there must be a means to sample the continuous flow associated with MD discretely into the analysis portion of the device. We have utilized PDMSbased pneumatic valves in bilayer microfluidic devices to interface continuous hydrodynamic flow and electroosmotic flow. ${ }^{15,16}$ When the valves are closed the two flows are separated. By actuating the valves, analyte from the continuously flowing sample stream is repetitively injected into a separation channel, where the analytes can be separated by electrophoresis.

While there have been recent reports from our group ${ }^{15}$ and others ${ }^{17}, 18$ of coupling MD with microchip electrophoresis (ME), all are done with fluorescence detection in irreversibly sealed devices. Our group has shown that a palladium decoupler can be used to integrate microchip electrophoresis and amperometric detection. ${ }^{19,}{ }^{20}$ The decoupler allows the electrodes to be placed directly in the electrophoresis channel, which minimizes the band broadening associated with end-channel detection where analyte bands diffuse as they transverse the distance between the channel outlet and the detection electrodes. ${ }^{21}$ This approach also enables the use of reversibly-sealed devices that avoid high temperature bonding steps and allow facile integration of carbon-based working electrodes. ${ }^{20}$

In this paper, we detail the development of a reversibly-sealed bilayer microfluidic device that enables the on-line coupling of MD sampling and ME with EC detection. The interface of the two flow regimes (MD/ME) is reproducibly fixed through lithography steps and the interface between ME and EC detection is realized by use of a palladium decoupler. We have optimized the fabrication and operation of the MD/ME/EC device and made it compatible for use with biological systems. We demonstrate the applicability of this device by monitoring the stimulated release of dopamine from PC 12 cells. This is the first known report of integrating on-line MD sampling and ME with EC detection. While this study is focused on in vitro applications, this same system can also be adapted for in vivo analysis in the future.

\section{Experimental}

\section{Microchip Fabrication}

Several individual fabrication procedures were required to develop the MD/ME/EC device that can couple the hydrodynamic flow associated with MD sampling to ME (using PDMS-based pneumatic valves) as well as integrate ME with EC detection (using a palladium decoupler and carbon ink microelectrodes).

Palladium Plate Fabrication-The Nanofabrication Facility at Stanford University was responsible for sputtering a titanium adhesion layer $(200 \AA)$ followed by a palladium layer (2000 ̊̊ or $4000 \AA$ ) on high quality, $100 \mathrm{~cm}$ diameter, borosilicate glass (Precision Glass and 
Optics, Santa Ana, CA). Photolithography with $A Z^{\circledR} 1518$ positive resist (AZ Resist, Somerville, NJ) and a positive photomask (3600 dpi, The Negative Image, St. Louis, MO) followed by wet etching procedures resulted in a palladium decoupler and dual electrode connectors (Figure 1). A previous study using a similar design showed that the optimum effective decoupler length to be $500 \mu \mathrm{m} .{ }^{19}$

Microelectrode Fabrication-The optimized procedure for producing dual carbon ink electrodes has been previously described. ${ }^{20}$ A micromolding structure offering channel dimensions of $13 \mu \mathrm{m}$ in length and $3.5 \mu \mathrm{m}$ in height was made by traditional soft photolithography techniques using SU-8 2 photoresist (Microchem, Newton, MA). Palladium connector electrodes facilitated an electrical connection to dual carbon ink microelectrodes as seen in Figure 1. The micromolding channels were aligned and reversibly sealed over the palladium connectors and an ink mixture was aspirated through the channels. Following two heating steps $\left(75^{\circ} \mathrm{C}, 1 \mathrm{hr}\right.$ and $\left.120^{\circ} \mathrm{C}, 1 \mathrm{hr}\right)$, the resulting carbon ink electrodes remained on the glass plate with a height of $1.1 \mu \mathrm{m}$ and a length of $13 \mu \mathrm{m}$. The optimal distance between the first electrode and the decoupler was previously found to be $250 \mu \mathrm{m}^{19}$ and the distance between the two electrodes has been optimized at $100 \mu \mathrm{m} .{ }^{20}$ Analytes were detected at the carbon electrode(s) using a 812 B potentiostat (CH Instruments, Austin, TX) set at one or two specific potentials depending on the use of single or dual working electrodes. All potentials throughout this paper are versus a platinum wire counter electrode.

Microchip Fabrication and Assembly-The creation of a bilayer device involved fabrication of both a flow layer and a valving layer and is outlined in Figure 1. A positive resist was used to create the flow channel master and a post-exposure bake was used to round the photoresist, which has proven to be crucial for proper valve actuation. ${ }^{22}$ The valving master was made with a negative resist. Pieces of PDMS were placed around the edge of the valving master to create a $6 \mathrm{~mm}$ tall mold to aid in the creation of a thick layer. To create the bilayer configuration of the microchip, the thick valving layer of PDMS was cut and removed from its mold. At this time, a 20-guage luer stub adapter was used to create inlet holes for the introduction of nitrogen gas needed for proper actuation of the valves. In addition, a $2 \mathrm{~mm}$ diameter hole was also manually removed using a circular razor from the portion of the thick layer that will later overlay the decoupler on the palladium plate as seen in Figure 1 (further detailed in results/discussion). Alignment of the valve layer onto the flow layer was done manually with the aide of a stereomicroscope. Upon sealing and curing of the two layers, a sample inlet was created through both layers using the luer stub adapter, while a $3 \mathrm{~mm}$ circular razor was used to form waste and buffer reservoirs. The chip was then aligned and reversibly sealed so that the hole created in the thick layer overlaid the decoupler (Figure 1). The separation channel was aligned so that it crossed only dual carbon ink electrodes without allowing the palladium electrode connectors to come in contact with the separation channel. The optimized chip design can be seen in Figure 2A. As mentioned above, the fluidic channels are rounded, with the hydrodynamic flow channel being $105 \mu \mathrm{m}$ in width and $22 \mu \mathrm{m}$ at maximum depth while the electrophoresis channel has the same depth but is $40 \mu \mathrm{m}$ in width. The distance from the sample inlet (S) to the valving interface is $1.5 \mathrm{~cm}$ and the effective separation length (distance from valving interface to the carbon ink detection electrodes) is 2.1 $\mathrm{cm}$.

\section{Chip Operation}

Dialysate from the MD probe was introduced to the microchip by directly inserting the commercially available MD tubing into the sample inlet hole (S) (Figures 2B and C). The PDMS-based pneumatic valves allowed flow to be hydrodynamically pumped through the flow portion of the microchip without being introduced into the separation channel. The valves were dead end filled with water through the use of pressurized water vials that were connected to 
solenoid valves (MAC Fluid Power Engineering, St. Louis, MO). PEEK tubing was connected to the water vials and steel pins were attached to the opposite end of the tubing and directly inserted in the valve access holes (Figure 2B). The pressure of the gas was controlled by a regulator and the solenoid valve was controlled by a power supply control unit (Instrument Design Lab, University of Kansas, Lawrence, KS). Once filled, the valves could be actuated as described below.

To initiate microchip electrophoresis-based separations, high voltage was applied to the buffer reservoir (B), a pushback voltage was applied to the pushback reservoir (PB), and a palladium decoupler served as a path to ground (Figure 1). A high voltage power source (Labsmith, Livermore, CA) offered a range of 0 to $+3000 \mathrm{~V}$ and also enabled a timed injection sequence during which the voltage could be turned off for a pre-determined amount of time. As described previously, the pushback voltages led to a small amount of electroosmotic flow down the pushback channel to minimize sample carryover at the injection interface as well as leakage into the electrophoresis separation channel. ${ }^{15}, 16$ In order to conduct an injection of sample, valve 2 (normally closed, NC) would open for a specified amount of time (actuation time), while valve 1 (normally open, NO) was concurrently closed. The dialysate was continuously pumped through the microchip and, as the valves were actuated, a discrete sample plug was injected into the separation channel. A typical injection volume used in these studies is 970 $\mathrm{pL}$. Separations occurred with a high voltage of $700 \mathrm{~V}$ applied to the buffer reservoir and 200 $\mathrm{V}$ applied to the pushback reservoir, creating a junction voltage ${ }^{23}$ of $427 \mathrm{~V}$. The electrophoresis buffer for all studies was $25 \mathrm{mM}$ boric acid with $3 \mathrm{mM}$ SDS (pH of 9.5). The electrophoresis buffer contained sodium dodecyl sulfate (SDS), as Culbertson's group has shown that the addition of SDS can eliminate analyte absorption as well as increase and stabilize the EOF. ${ }^{24}$

\section{Microdialysis experiments}

During the characterization stages of the microdialysis experiments, a Bioanalytical Systems (BAS, West Lafayette, IN) brain probe was used (either $2 \mathrm{~mm}$ or $4 \mathrm{~mm}$ membrane). A $5 \mathrm{~mm}$ membrane linear probe (BAS) was used for the concentration change and cell experiments. A gastight syringe was used to precondition the probes by first flushing with water for 10-20 minutes and then flushing with the appropriate perfusate buffer. For the brain probe experiments, a $38-\mathrm{cm}$ segment of FEP tubing $(650 \mu \mathrm{m}$ o.d. $\times 120 \mu \mathrm{m}$ i.d. $)$ was connected from a $250 \mu \mathrm{L}$ gastight syringe to the probe. The probe itself had a polyacrylonitrile (PAN) membrane with a $<5000 \mathrm{Da}$ molecular weight cutoff and a volume of $2.3 \mu \mathrm{L}$ (as reported by BAS). Sample exiting the probe was carried to the microchip using a 12-cm segment of FEP tubing (793 $\mu \mathrm{m}$ o.d. $\times 76 \mu \mathrm{m}$ i.d.), with the tubing being directly inserted into the inlet of the chip. A 5 mm PAN membrane with < 5000 Da molecular weight cutoff was used in the linear probe experiments. Linear probes are commercially manufactured with the proper tubing (180 $\mu \mathrm{m}$ i.d.) directly connected to the membrane. A $2.7-\mathrm{cm}$ segment of tubing ran from the membrane and, in order to minimize dead volume, this tubing was then connected to $50 \mu \mathrm{m}$ i.d. ( $350 \mu \mathrm{m}$ o.d.) capillary that was $7 \mathrm{~cm}$ in length. The opposite end of the capillary was fitted with a $794 \mu \mathrm{m}$ o.d. capillary tubing sleeve (Upchurch Scientific) and inserted directly into the microchip inlet. The dead volume associated with the probe, microdialysis tubing, connecting capillary, inlet hole, and the sample channel was $\sim 1.65 \mu \mathrm{L}$. However, once the sample solution is transferred to the chip, the dead volume is minimal and the time it takes sample to travel from the inlet to the interface and then to the electrodes for detection is $\sim 14 \mathrm{sec}$. The stated analyte concentrations correspond to the solution to which the probe is exposed.

\section{In Vitro Experiments}

If not stated otherwise, chemicals were purchased from Sigma-Aldrich (St. Louis, MO). For cell studies, untreated $35 \mathrm{~mm}$ Petri dishes (Fisher Scientific, Springfield, NJ) were coated with polygen, a solution of $60.0 \mu \mathrm{L}$ of PureCol collagen solution (Inanmed, Fremont, CA), $4.5 \mathrm{~mL}$ 
of a $0.03 \mathrm{mg} / \mathrm{mL}$ poly-1-lysine solution, and $9.0 \mathrm{~mL}$ of $30 \%$ ethanol. The polygen was then left in the dishes for one hour, removed, and allowed to dry overnight, after which the flasks were rinsed with 10X phosphate buffered saline solution (Fisher Scientific, Springfield, NJ) and then replaced by F-12K medium (Kaighn's modification of Ham's F-12 medium; ATCC, Manassas, Virginia) supplemented with $10 \%$ penicillin-streptomycin solution, $5 \%$ fetal bovine serum (ATCC), and 10\% horse serum (ATCC). PC 12 cells (ATCC) were then transferred into the Petri dishes and placed into the incubator at $37^{\circ} \mathrm{C}$ and $7 \% \mathrm{CO}_{2}$. Cell medium was replaced every 1- 2 days and sub-cultured as needed (approximately 7 days).

PC 12 cells were allowed to grow until 90\% confluency prior to analysis. The cells were preloaded one hour preceding analysis by exposing them to $1 \mathrm{mM}$ dopamine. ${ }^{25}$ Media and dopamine were removed from the cells prior to analysis and rinsed three times with a cell compatible buffer to ensure full removal of media and residual dopamine from the cells. The cell compatible buffer (pH 7.4) was made up of the following: $1.4 \mathrm{mM} \mathrm{KCl}, 72.5 \mathrm{mM} \mathrm{NaCl}$, $0.5 \mathrm{mM} \mathrm{MgCl}_{2}, 0.6 \mathrm{mM} \mathrm{CaCl}_{2}$, and $0.25 \mathrm{mM} \mathrm{NaH}_{2} \mathrm{PO}_{4}$. Blank runs were performed by exposing the cells to only this buffer. Cells were then exposed to $1 \mathrm{~mL}$ of $\mathrm{K}^{+} / \mathrm{Ca}^{2+}$ stimulant solution. The stimulant solution ( $\mathrm{pH}$ 7.4) was comprised of $80 \mathrm{mM} \mathrm{KCl}, 150 \mathrm{mM} \mathrm{NaCl}, 0.7$ $\mathrm{mM} \mathrm{MgCl}_{2}, 2 \mathrm{mM} \mathrm{CaCl}_{2}$, and $1 \mathrm{mM} \mathrm{NaH}_{2} \mathrm{PO}_{4}$, and $10 \mathrm{mM} \mathrm{HEPES}{ }^{26}$ The probe was perfused with a solution of the cell compatible buffer containing $3 \mathrm{mM}$ sodium dodecyl sulfate (SDS). Sampling occurred at a perfusate flow rate of $0.3 \mu \mathrm{L} / \mathrm{min}$ and EC detection took place at +0.9 $\mathrm{V}$ at a single electrode with a high voltage and pushback voltage of $700 \mathrm{~V}$ and $200 \mathrm{~V}$, respectively. Injections occurred approximately every $20 \mathrm{sec}$ with a $900 \mathrm{msec}$ valve actuation time and $2 \mathrm{sec}$ high voltage shutoff (discussed in results/discussion). Cells were counted after stimulation with a hemocytometer. Dopamine standards were prepared daily, sampled through the same probe, and analyzed using the same MD/ME/EC device.

\section{Results and Discussion}

\section{Integration of Microdialysis Sampling with Microchip Electrophoresis/Electrochemistry}

The only previous example of the on-line coupling of MD sampling with conventional CE and EC detection has been performed by Lunte's group at the University of Kansas. ${ }^{11}$ The set-up consists of a gap junction interface and a fracture-type decoupler. However, this coupling has not been commercialized due to the difficulty associated with integrating and aligning all of the various components. Two different couplings must occur in order to integrate MD with $\mathrm{ME}$ and EC detection. The hydrodynamic flow from the MD probe must be coupled with the electrophoretic flow characteristic of ME and the high voltages associated with ME must be isolated from the EC detection scheme. It is shown in this work that microchip-based analysis systems enable the integration of these otherwise separate processes in a manner where the interface is fixed and reproducible.

Previous work from our lab showed that continuous hydrodynamic flow could be coupled to electrophoresis with the use of PDMS-based pneumatic valves. ${ }^{15}$, 16 However, those studies were conducted with direct infusion of sample and fluorescence detection and our goal here is to couple MD sampling and ME with EC detection for the direct detection of catecholamines. A similar approach was used here to couple MD sampling and ME, with the interface between the dialysate and the electrophoresis domains being the PDMS-based pneumatic valves (Figure 2A). The PDMS-based valving interface actuates on the order of hundreds of milliseconds to discretely inject dialysate into the electrophoresis portion of the chip. A previous study showed that two valves were needed in the system (as opposed to just using valve \#2) in order to increase reproducibility. ${ }^{16}$ The procedure used to fabricate the reversibly sealed device is shown in Figure 1. After full curing in an oven, the chip can be reversibly sealed to a glass plate. The ability to perform a reversible seal is in part due to the capability of the $\sim 3.5 \mathrm{~mm}$ thick PDMS chip to support the tubing and steel pins used to introduce fluids. A reversible seal is 
advantageous, as it avoids high temperature bonding steps that can limit electrode lifetime, enables facile integration of carbon-based working electrodes, and makes the microchip conducive to cleaning so that costly electrode plates can be reused. ${ }^{20}$ As detailed in the experimental section, by matching the outer diameter of the tubing coming from the probe with the dimensions of the inlet hole, the tubing could be simply inserted into the microchip so that the fluidic connection was extremely straightforward (Figures $2 \mathrm{~B}$ and $\mathrm{C}$ ).

While using the PDMS-based pneumatic valves makes the fluidic coupling relatively simple, integration of the MD/ME system with EC detection required extensive optimization. We have previously shown the ability to reproducibly align and pattern dual carbon ink microelectrodes with a palladium decoupler for microchip-based electrophoresis studies. ${ }^{20}$ The decoupler absorbs hydrogen produced at the electrophoretic ground ensuring a field free region downstream. The detection electrodes are effectively isolated from the electric field while remaining in the separation channel to minimize band broadening. ${ }^{19}$ In order to create a palladium decoupler on glass, traditional sputtering and photolithography procedures were employed. Carbon ink microelectrodes were patterned over palladium connectors to be 1.1 $\mu \mathrm{m}$ in height and $13 \mu \mathrm{m}$ in length. ${ }^{20}$ The steps required to integrate EC detection with MD sampling and ME are detailed below. For the initial characterization studies, a brain probe (2 or $4 \mathrm{~mm}$ membrane) was utilized.

Thick Layer Removal-Quake's group has shown that the thickness of the valving layer is needed to support the weight of the steel pins inserted into the chip to actuate the valves. ${ }^{22}$ However, when the thick bilayer microchip was reversibly sealed to the glass plate with palladium decoupler and carbon ink electrodes, several issues arose. Because the decoupler acts as a cathode in this system, hydrogen is produced at the decoupler. ${ }^{19}$ Previous work with a simple gated microchip (about $1 \mathrm{~mm}$ thick) showed that the hydrogen produced effectively diffused through the gas-permeable PDMS. ${ }^{20}$ However, with a $3.5 \mathrm{~mm}$ thick microchip the hydrogen could not effectively diffuse through the PDMS. This caused the formation of hydrogen gas bubbles in the separation channel and ceased the separation. To overcome this limitation, a portion of the thick layer that overlaid the decoupler was removed during the fabrication process. As seen in Figure 1, before the thick layer is sealed to the thin flow layer, a $2 \mathrm{~mm}$ circular razor was used to remove a portion of the thick layer. When aligned, this hole seals over the decoupler. In this way, the hydrogen produced at the decoupler only had to diffuse through the thin flow layer $\left(\sim 40 \mu \mathrm{m}^{22}\right)$. With the removal of the thick layer over the decoupler, separations were run with field strengths as high as $800 \mathrm{~V} / \mathrm{cm}$ and showed linear Ohm's Law plots (data not shown), whereas without this modification, separations were performed at field strengths no higher than $300 \mathrm{~V} / \mathrm{cm}$.

To demonstrate the MD/ME/EC integration, a MD brain probe with a $2 \mathrm{~mm}$ membrane was used to sample dopamine and catechol standards from a $35 \mathrm{~mm}$ Petri dish. The perfusate and electrophoresis buffers were matched by using a $25 \mathrm{mM}$ boric acid/3 mM SDS buffer. The sample analytes diffused across the membrane and were swept out as dialysate and pumped directly into the sample inlet of the bilayer device. Discrete plugs of the sample were then injected in the separation channel by actuating valves for a given amount of time. A high voltage of $700 \mathrm{~V}$ and pushback voltage of $200 \mathrm{~V}$ were used to separate the analytes, which were subsequently detected at carbon ink microelectrodes. As seen in Figure 3, not only single electrodes, but also dual electrodes can be used with this scheme to monitor reversible redox systems. This electropherogram shows both the oxidative $(+0.9 \mathrm{~V})$ and reductive $(+0.1 \mathrm{~V})$ traces for the separation achieved between dopamine and catechol (1 mM each) as sampled by the probe at a perfusate flow rate of $1 \mu \mathrm{L} / \mathrm{min}$ with $500 \mathrm{msec}$ valve actuation. With $n=11$, the average peak height RSD for analyzing dopamine and catechol were $5.68 \%$ and $5.29 \%$, respectively. 
Buffers-Although the previous separation shows the on-line integration of MD sampling, $\mathrm{ME}$, and dual electrode amperometric detection, the buffer used is not compatible with biological systems. Buffers used in biological systems are relatively high in ionic strength and commonly contain numerous salts with the sum salt concentration being on the order of 100 $\mathrm{mM}$. To make the MD/ME/EC system useful for studying biological systems, the perfusate buffer was changed to a cell compatible buffer whose composition consisted of $1.4 \mathrm{mM} \mathrm{KCl}$, $72.5 \mathrm{mM} \mathrm{NaCl}, 0.5 \mathrm{mM} \mathrm{MgCl}_{2}, 0.6 \mathrm{mM} \mathrm{CaCl}_{2}$, and $0.25 \mathrm{mM} \mathrm{NaH}_{2} \mathrm{PO}_{4}$ at a pH of 7.4. Samples were placed in the cell compatible buffer and sampled with similar perfusate. Once sample diluted with the cell compatible buffer was injected into the separation channel, bubbles were produced at the decoupler, inhibiting the electrophoresis. The salt content of the cell compatible buffer increased the overall current in the separation channel to such an extent that the resulting increase in hydrogen production could no longer be dissipated by the decoupler. Therefore, optimization of the decoupler was explored to enable the injection of high ionic strength samples.

Decoupler Size-The initial decoupler size was $500 \mu \mathrm{m}$ in length, $2000 \AA$ in height and the exposed width was defined by the channel width $(40 \mu \mathrm{m}$, see Figure 1). Initially, the field strength was decreased to $280 \mathrm{~V} / \mathrm{cm}$ and while this helped decrease bubble formation, a separation sequence could not continue longer than ten minutes before hydrogen bubble formation occurred at the decoupler. To further overcome this issue, the effect of surface area on the ability to dissipate the hydrogen produced at the palladium decoupler was investigated. First, the decoupler height was increased to $4000 \AA$ A. Decoupler lengths of $0.5,1,1.5,2$, and 3 $\mathrm{mm}$ were utilized and it was found that a length of at least $2 \mathrm{~mm}$ was necessary for continued operation of the decoupler with the high salt content samples. It was also found that the use of a $2 \mathrm{~mm}$ decoupler that was $2000 \AA$ in height did not function for an extended period of time; therefore, the decoupler height and subsequent edge plane effects are important but the decoupler length also plays a role in increasing the surface area so that the decoupler can effectively dissipate the current and hydrogen produced from the separation voltage. In previous studies, we showed that a small amount of band broadening does occur after the separation is grounded as a result of parabolic flow after the decoupler. ${ }^{19},{ }^{20}$ In addition, since the cell compatible buffers have a high salt content and they are being injected into a separation buffer of considerably lower ionic strength, there is significant amount of plug destacking ${ }^{27}$ occurring during the electrophoretic separation. However, as seen in Figure 4, even with the increased decoupler length (with a corresponding increased distance for band broadening before detection) and plug destacking a separation between dopamine, norepinephrine, and ascorbic acid was possible, with plate numbers of 34,000 plates $/ \mathrm{m}$ for norepinephrine.

High Voltage Shutoff-An additional adjustment to decrease the electrophoretic current is to shut off the high voltage while an injection is made. During the short period of time that the injection valve is open, the high voltage is exposed to the relatively wide $(105 \mu \mathrm{m})$ flow channel that contains the high ionic strength cell compatible buffer. This, in turn, increased the electrophoresis current during an injection and immediately caused bubble formation at the decoupler. To circumvent this, an injection sequence was developed in which the high voltage is programmed to shut off for $2 \mathrm{sec}$. During this time, the valves were actuated, which allowed a plug to be injected into the separation channel. Once injected, the high voltage resumed and the separation was initiated. With all adjustments to the system described above, separations were capable of continuing for the extent of all experiments without any hydrogen bubble formation. Figure 4 shows the separation of dopamine, norepinephrine, and ascorbic acid with the optimized decoupler size and voltage shutoff sequence. Samples were made in cell compatible buffer and sampled through the MD probe at a perfusate flow rate of $0.3 \mu \mathrm{L} / \mathrm{min}$. The field strength remained at $280 \mathrm{~V} / \mathrm{cm}$ and the oxidative potential was set to $+0.9 \mathrm{~V}$. 


\section{Concentration Change Experiment}

In order to evaluate how well the device responds to external changes in concentration, a study monitoring an increase in dopamine concentration as sampled through a linear MD probe was performed. As described below, we also use this device to monitor the release of dopamine from a layer of PC 12 cells. Utilizing a linear probe with a $5 \mathrm{~mm}$ membrane allowed us to minimize the dilution of cell release by decreasing the total volume of buffer in the Petri dish. The brain probe was $4 \mathrm{~mm}$ in length and placing it perpendicular to the dish surface required at least $2 \mathrm{~mL}$ of solution to fully cover the exposed membrane. With the linear probe, the required solution volume was decreased to $1 \mathrm{~mL}$, as the probe simply placed across the bottom of the dish and needed little solution to fully submerge the membrane. Using the linear probe and dopamine as the analyte at a perfusate flow rate of $0.3 \mu \mathrm{L} / \mathrm{min}$, a $77 \%$ recovery and $9 \mu \mathrm{M}$ limit of detection limit was found. When the recovery is considered, this limit of detection is consistent with our previous work using these electrodes with a palladium decoupler and ME, ${ }^{20}$ although other approaches have resulted in lower limits of detection. ${ }^{28}$

Monitoring off-chip changes in concentration are important in the coupling of a ME/EC system to off-chip processes such as MD sampling, where the temporal resolution is of importance. Lag and rise time are commonly used to evaluate these systems. Lag time is defined by the amount of time needed for the device to start to respond to a change in concentration. The rise time, or temporal resolution, is defined as the time during which signals increase from $10 \%$ to $90 \%$ of the maximum intensity. ${ }^{8,29}$ To characterize the system and monitor the ability of the device to monitor a change in dopamine concentration, a $5 \mathrm{~mm}$ linear membrane probe was used. In order to minimize the tubing dead volume, the tubing exiting the commercially available probe ( $2.7 \mathrm{~cm}$ in length, $180 \mu \mathrm{m}$ i.d.) was transitioned to a $50 \mu \mathrm{m}$ i.d. ( $350 \mu \mathrm{m}$ o.d.) capillary that was $7 \mathrm{~cm}$ in length. The opposite end of this capillary was fitted with a capillary sleeve that enabled the tubing to be directly inserted into the microchip inlet.

The results of concentration change experiment are shown in Figure 5. Prior to the experiment, the probe was allowed to equilibrate. Initially, cell compatible buffer containing $100 \mu \mathrm{M}$ dopamine was placed in a $35 \mathrm{~mm}$ Petri dish and the probe was perfused with cell compatible buffer containing $3 \mathrm{mM}$ SDS (pH 7.4). Several injections were made in order to achieve a baseline response. With the continual sampling, injection, and separation occurring, an aliquot of a dopamine standard solution was delivered to the Petri dish so that the final dopamine concentration was $300 \mu \mathrm{M}$. The valves were actuated for a period of $400 \mathrm{msec}$ during a programmed period of high voltage shutoff and this actuation was repeated every $20 \mathrm{sec}$. A perfusate flow rate of $0.3 \mu \mathrm{L} / \mathrm{min}$ was used and voltages of $700 / 200 \mathrm{~V}$ were applied to the buffer and pushback reservoir, respectively, with amperometric detection at $+0.9 \mathrm{~V}$ using a single carbon ink electrode. This study operated at low flow rates to ensure a high recovery across the probe (addressed above). As shown in Figure 5, the lag and rise times for the dopamine concentration change study were $330 \mathrm{sec}$ and $150 \mathrm{sec}$, respectively. Taking into account the associated probe, tubing, connector dead volumes (see experimental), and separation time leads to a theoretical lag time value of $337 \mathrm{sec}$. These results are very comparable to previous work where microdialysis was integrated with microchip electrophoresis and fluorescence detection. ${ }^{17}, 18$ To decrease external dead volume and lag times, it is possible to create probes with lower volume and connector tubing that is minimal in length and inner diameter. ${ }^{7,}, 9,10$ In addition, Norton et al. recently showed that the temporal resolution of microdialysis sampling is limited by Taylor dispersion in the probe outlet tubing. Kennedy's lab also recently detailed an approach that uses chip-based segmented flow analysis using oil/water droplets to limit the amount of this dispersion. When this is done, rise times as short as $15 \mathrm{sec}$. are possible, although lag times are still limited by the time to transverse the connector tubing and other system volumes. 


\section{In Vitro Analysis}

To demonstrate the ability of the MD/ME/EC system to monitor a biological system, PC 12 cells were grown in a $35 \mathrm{~mm}$ Petri dish and the stimulated release of dopamine was sampled through a linear probe. Figure 6A shows a micrograph of the PC 12 cells during an experiment where the linear probe is sampling the stimulated release of dopamine. Before the experiment was carried out, media was removed from the cells and they were rinsed three times with cell compatible buffer. The cells were then exposed to cell compatible buffer and sampled with the linear MD probe. Figure 6 shows a baseline response that results from sampling the cell compatible buffer prior to cell stimulation. To initiate cell release, the cell compatible buffer was removed from the dish and replaced with $1 \mathrm{~mL}$ of a $\mathrm{K}^{+} / \mathrm{Ca}^{2+}$ stimulant solution. This solution was continually sampled by MD, injected, separated, and amperometrically detected at $+0.9 \mathrm{~V}$. After analysis, dopamine standards were sampled with the same probe to determine the concentration of dopamine released and to confirm the presence of dopamine by matching the migration time (data not shown). The data shown in Figure 6B corresponds to an average dopamine concentration of $29.6 \mu \mathrm{M}$. Based on the number of cells (counted by hemocytometer), it was determined that there was $\sim 5.5$ fmole of dopamine released per cell. This is an approximate number, as there is some error associated with the counting of cells. In addition, the length of the experiment ( $20 \mathrm{~min}$.) could lead to reuptake of the released dopamine. Nevertheless, this study shows that the fully integrated device can be used to selectively quantitate analytes sampled from a biological system.

\section{Conclusion}

These studies have shown that microchip technology can be used to integrate microdialysis sampling with electrophoresis and electrochemical detection. A bilayer PDMS device with pneumatic valves offered a means for coupling the continuous hydrodynamic flow from the microdialysis probe with electrophoretic flow. The additional integration of microdialysis sampling and microchip electrophoresis with electrochemistry enabled the detection of multiple analytes at single or dual carbon microelectrodes. In addition, all components were reproducibly aligned in fixed positions. While the salt content of biological buffers proved to be a challenge when working with the decoupler and amperometric detection, multiple modifications led to the successful monitoring of a biological system with the MD/ME/EC device. To the best of our knowledge, this is the first microchip-based system coupling microdialysis sampling and microchip electrophoresis with electrochemical detection. Although the device was used to monitor the stimulated release of dopamine from PC 12 cells, the MD/ME/EC device could be used as a general analytical tool to study other cell lines or for in vivo studies. The ability to utilize an electrophoresis-based separation with electrochemical detection will be a benefit when trying to quantitate neurotransmitters that have been sampled by microdialysis but are unable to be differentiated by electrochemistry alone (such as dopamine and norepinephrine). ${ }^{30,}{ }^{31}$ Future work will detail studies to decrease the lag and rise times as well improve the limits of detection.

\section{Acknowledgments}

This research was supported by a grant from the National Institutes of Health (9R15GM084470-02).

\section{References}

1. Davies MI, Cooper JD, Desmond SS, Lunte CE, Lunte SM. Adv Drug Deliv Rev 2000;45:169-188. [PubMed: 11108973]

2. Ungerstedt, U. Measurement of Neurotransmitter Release In Vivo. John Wiley \& Sons Ltd.; New York: 1984.

3. Dawson LA, Stow JM, Palmer AM. J Chromatogr 1997;694:455-460. 
4. Kostel KL, Lunte SM. J Chromatogr 1997;695:27-38.

5. Qian J, Wu Y, Ynag H, Michael AC. Anal Chem 1999;71:4486-4492. [PubMed: 10546530]

6. Robert F, Bert L, Danoroy L, Renaud B. Anal Chem 1995;67:1838-1844. [PubMed: 9306734]

7. Lada MW, Vickroy TW, Kennedy RT. Anal Chem 1997;69:4560-4565. [PubMed: 9375517]

8. Wang M, Roman GT, Schultz K, Jennings C, Kennedy RT. Anal Chem 2008;80:5607-5615. [PubMed: 18547059]

9. Bowser MT, Kennedy RT. Electrophoresis 2001;22:3668-3676. [PubMed: 11699904]

10. Lada M, Kennedy RT. Anal Chem 1996;68:2790-2797. [PubMed: 8794915]

11. Zhou J, Heckert DM, Zuo H, Lunte CE, Lunte SM. Anal Chim Acta 1999;379:307-317.

12. Hogan BL, Lunte SM, Stobaugh JF, Lunte CE. Anal Chem 1994;66:596-602. [PubMed: 8154588]

13. Anderson BB, Ewing AE. J Pharm Biomed Anal 1999;19:15-32. [PubMed: 10698565]

14. Martin, RS. Methods in Molecular Biology, vol 339: Microchip Capillary Electrophoresis: Methods and Protocols. Henry, CS., editor. Humana Press; Towtowa, NJ: 2006.

15. Li MW, Huynh BH, Hulvey MK, Lunte SM, Martin RS. Anal Chem 2006;78:1042-1051. [PubMed: 16478094]

16. Li MW, Martin RS. Electrophoresis 2007;28:2478-2488. [PubMed: 17577199]

17. Huynh BH, Fogarty BA, Martin RS, Lunte SM. Anal Chem 2004;76:6440-6447. [PubMed: 15516139]

18. Sandlin ZD, Shou M, Shackman JG, Kennedy RT. Anal Chem 2005;77:7702-7708. [PubMed: 16316179]

19. Lacher NA, Lunte SM, Martin RS. Anal Chem 2004;76:2482-2491. [PubMed: 15117187]

20. Mecker LC, Martin RS. Electrophoresis 2006;27:5032-5042. [PubMed: 17096314]

21. Martin RS, Ratzlaff KL, Huynh BH, Lunte SM. Anal Chem 2002;74:1136-1143. [PubMed: 11924975]

22. Unger MA, Chou HP, Thorsen T, Scherer A, Quake SR. Science 2000;288:113-116. [PubMed: 10753110]

23. Seiler K, Fan ZHH, Fluri K, Harrison DJ. Anal Chem 1994;66:3485-3491.

24. Roman GT, McDonald K, Culbertson CT. Analyst 2006;131:195-201.

25. Moore JM, Papke JB, Cahill AL, Harkins AB. Am J Physiol Cell Physiol 2006;291:C270-281. [PubMed: 16467400]

26. Kozminski KD, Gutman DA, Davila V, Sulzer D, Ewing AG. Anal Chem 1998;70:3123-3130. [PubMed: 11013717]

27. Zhao Y, Lunte CE. Anal Chem 1999;71:3985-3991. [PubMed: 10500485]

28. Vickers JA, Henry CS. Electrophoresis 2005;26:4641-4647. [PubMed: 16294295]

29. Skoog, DA.; Holler, FJ.; Nieman, TA. Principles of Instrumental Analysis. Vol. 6th. Brooks/Cole; 1998.

30. Heien ML, Philips PEM, Stuber GD, Seipel AT, Wightman RM. Analyst 2003;128:1413-1419. [PubMed: 14737224]

31. Michael DJ, Wightman RM. J Pharm Biomed Anal 1999;19:33-46. [PubMed: 10698566] 


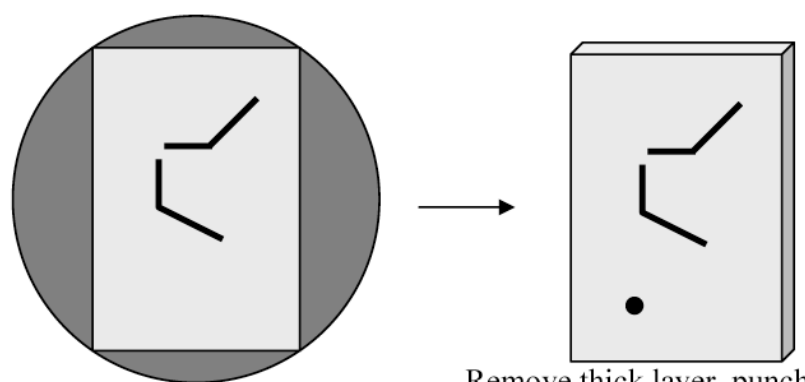

Pour 5:1 PDMS thick layer

Remove thick layer, punch access holes, and create hole where chip

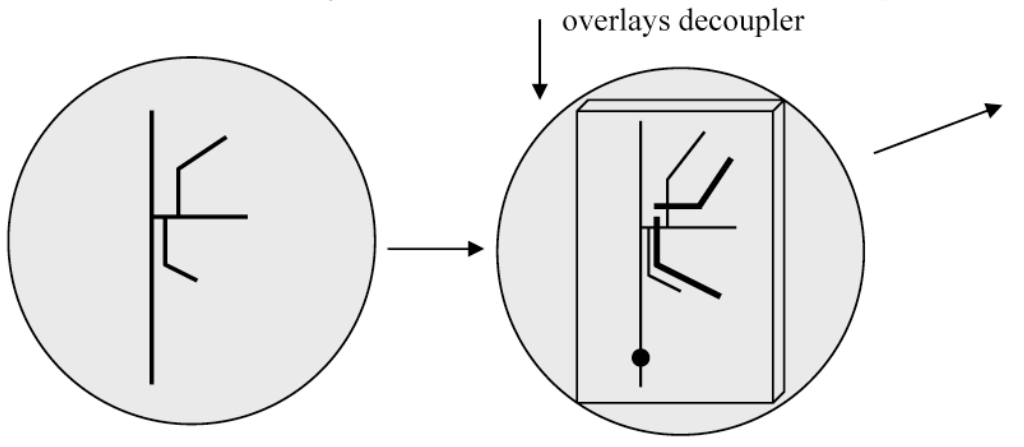

Align and seal thick layer onto

Spin-coat 20:1 PDMS thin layer thin layer and bake 1 hour

Pd electrode Decoupler connectors

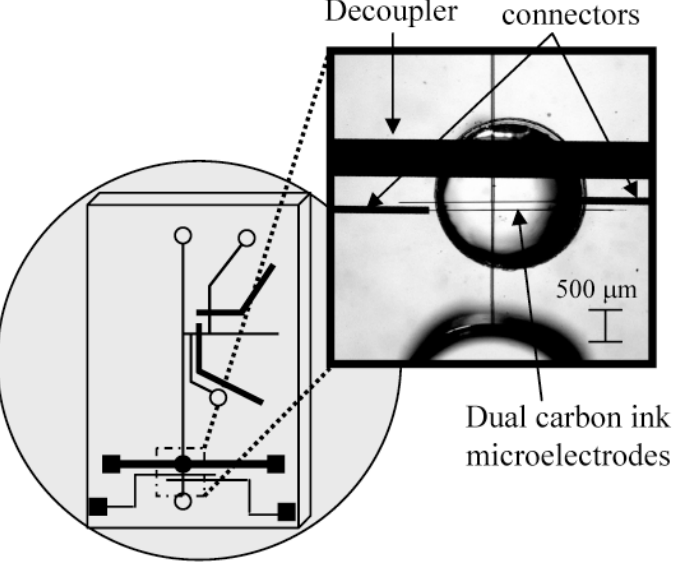

Remove bilayer chip and reversibly seal to Pd plate with carbon ink electrodes

Figure 1.

Fabrication of bilayer valving microchip. PDMS valving and flow layers were produced individually (both at $75^{\circ} \mathrm{C}$, valve layer for $20 \mathrm{~min}$ and flow layer for $15 \mathrm{~min}$ ) and then cured together as one uniform bilayer device (for $60 \mathrm{~min}$ at $75^{\circ} \mathrm{C}$ ). The microchip was then aligned and reversibly sealed onto the palladium glass plate. Micrograph shows a close-up view of decoupler where the thick layer has been removed and aligned over the decoupler and carbon ink microelectrodes. 

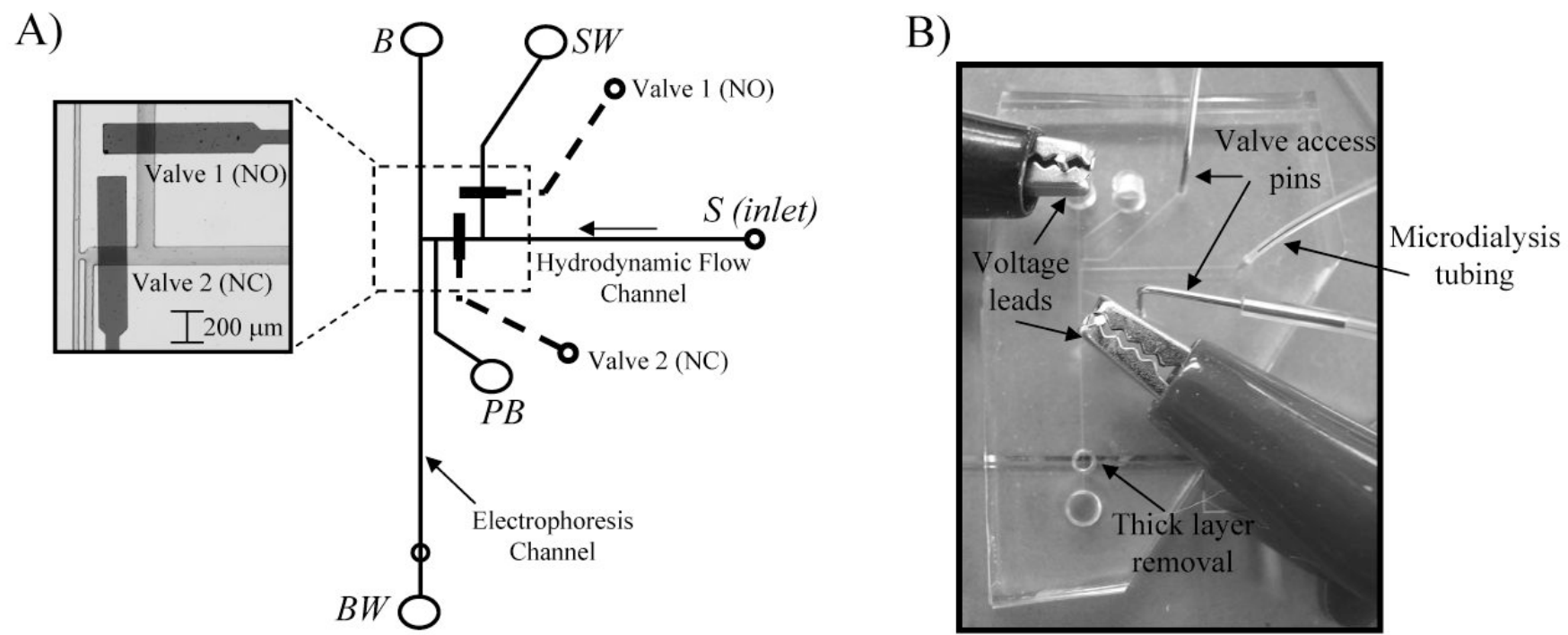

C)

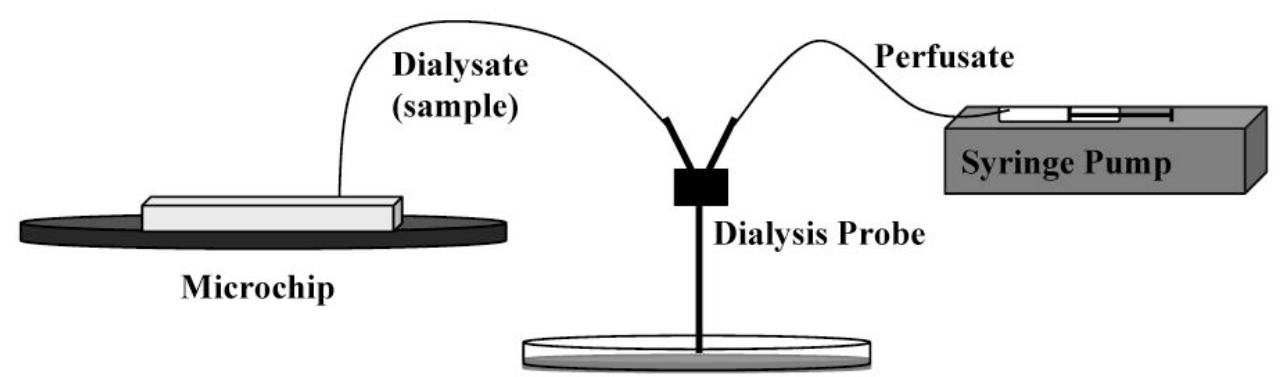

System to be sampled

Figure 2.

A) Schematic of bilayer microchip with picture of valving interface between hydrodynamic flow and electrophoretic flow. Abbreviations: B: buffer; BW: buffer waste; PB: pushback; SW: sample waste; S: sample; NO: normally open; NC: normally closed. B) Picture taken on grey background showing how the microdialysis tubing, access pins, and voltage leads are inserted into the microchip. C) Coupling of the microchip device with microdialysis sampling, associated tubing, and syringe pump. 


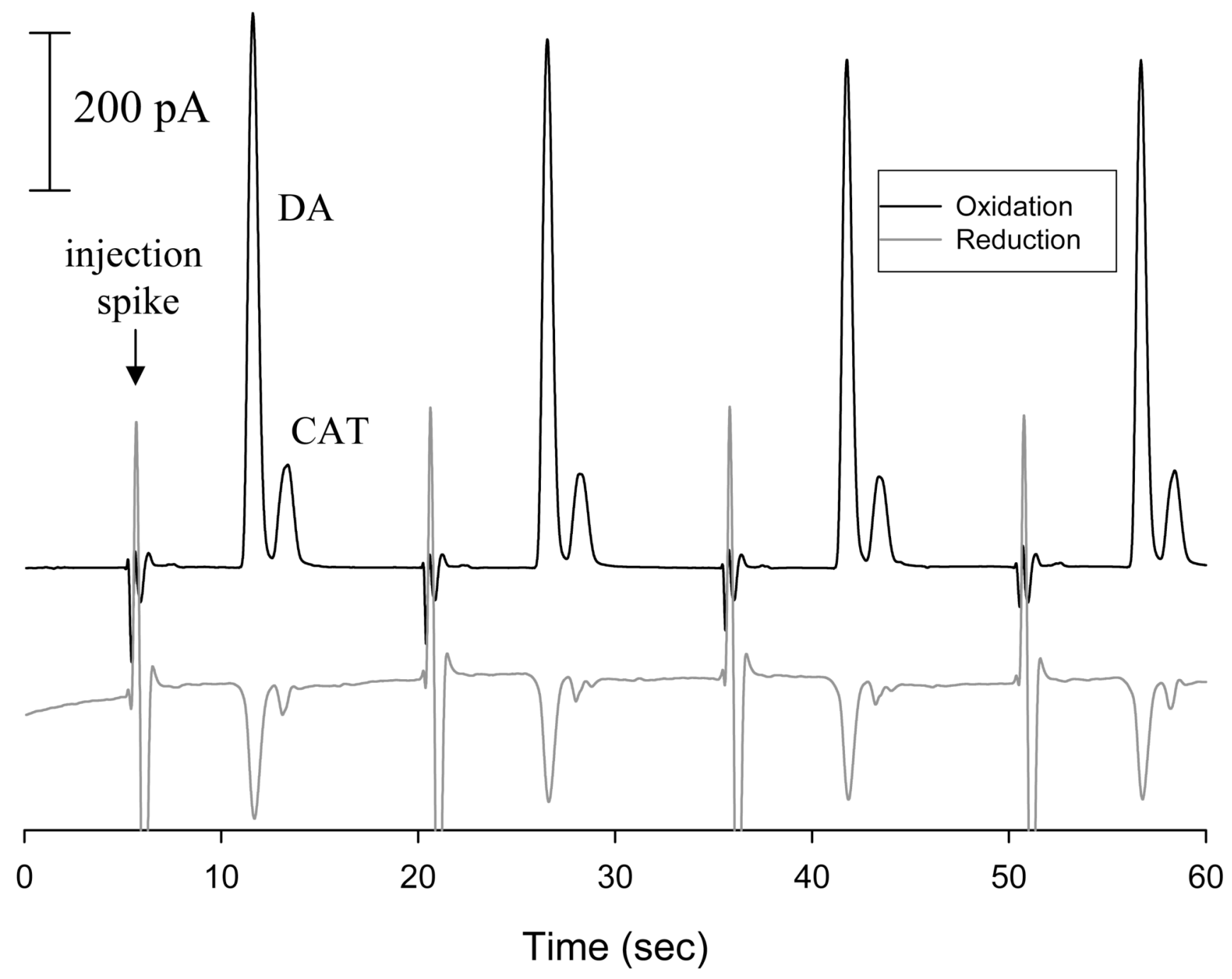

Figure 3.

Electrophoresis separation with dual electrode amperometric detection of dopamine (DA) and catechol (CAT) as sampled by microdialysis and discretely injected into the separation channel using pneumatic valves. The separation occurred with high voltage/pushback voltage of 700 $\mathrm{V} / 200 \mathrm{~V}$ and both oxidative $(+0.9 \mathrm{~V})$ and reductive $(+0.1 \mathrm{~V})$ traces are shown. Microdialysis sampling performed at a perfusate flow rate of $1.0 \mu \mathrm{L} / \mathrm{min}$ using brain probe with $2 \mathrm{~mm}$ membrane and $500 \mathrm{msec}$ valve actuation. $25 \mathrm{mM}$ boric acid/3 mM SDS buffer (pH 9.5) served as perfusate, sampling buffer, and run buffer. Analyte concentrations $=1 \mathrm{mM}$. 


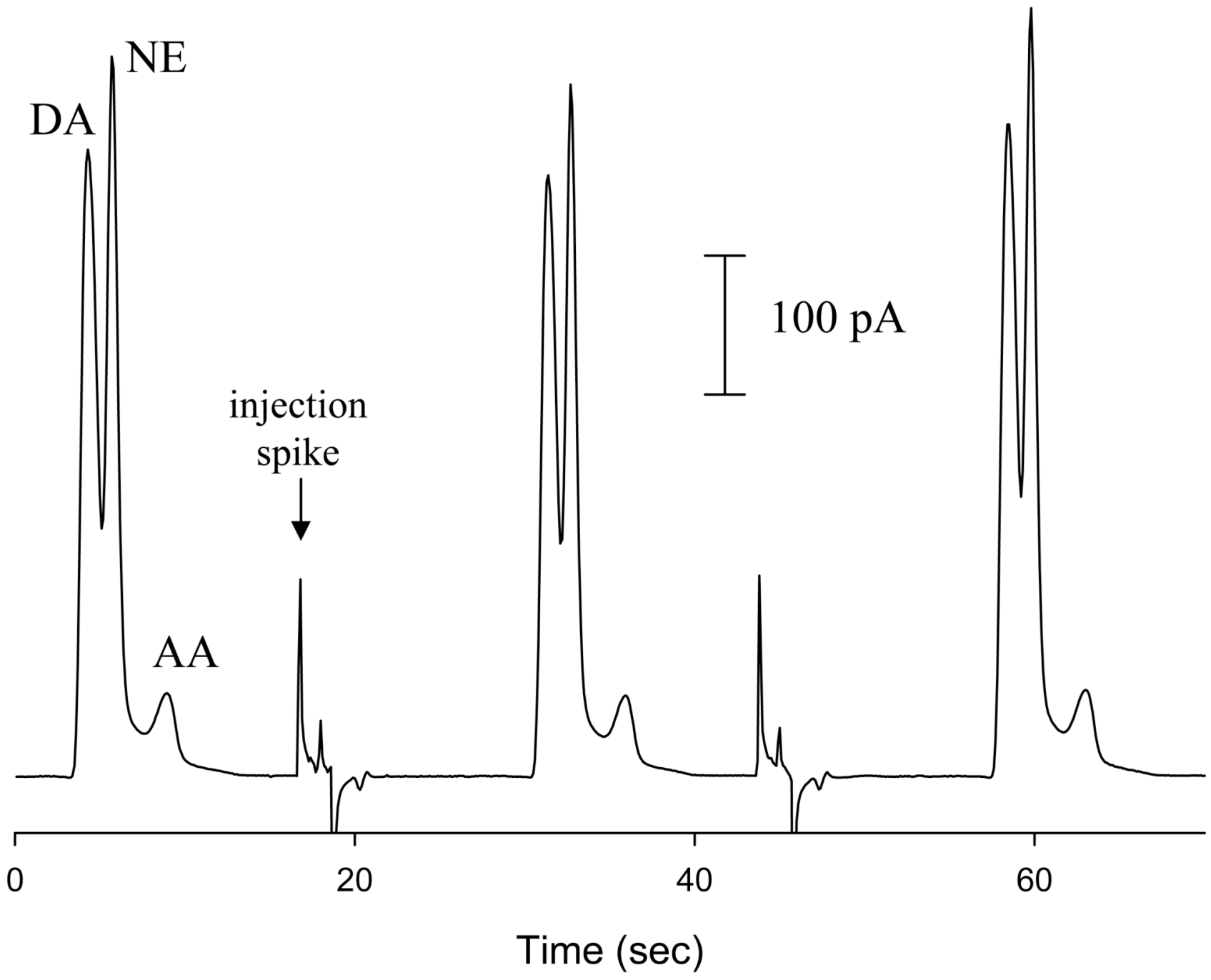

Figure 4.

Separation and detection (+ $0.9 \mathrm{~V})$ of dopamine (DA, $1 \mathrm{mM})$, norepinephrine $(\mathrm{NE}, 1 \mathrm{mM})$, and ascorbic acid (AA, $2 \mathrm{mM}$ ) as analyzed by the MD/ME/EC system using a $4 \mathrm{~mm}$ membrane brain probe and biologically compatible buffer. Perfusate flow rate of $0.3 \mu \mathrm{L} / \mathrm{min}$ with 700 $\mathrm{V} / 200 \mathrm{~V}$ applied to buffer/pushback channels. Electrophoresis buffer $=25 \mathrm{mM}$ boric acid with $3 \mathrm{mM}$ SDS (pH 9.5). Injection sequence: $1.1 \mathrm{sec}$ valve actuation with $2 \mathrm{sec}$ high voltage shutoff. 


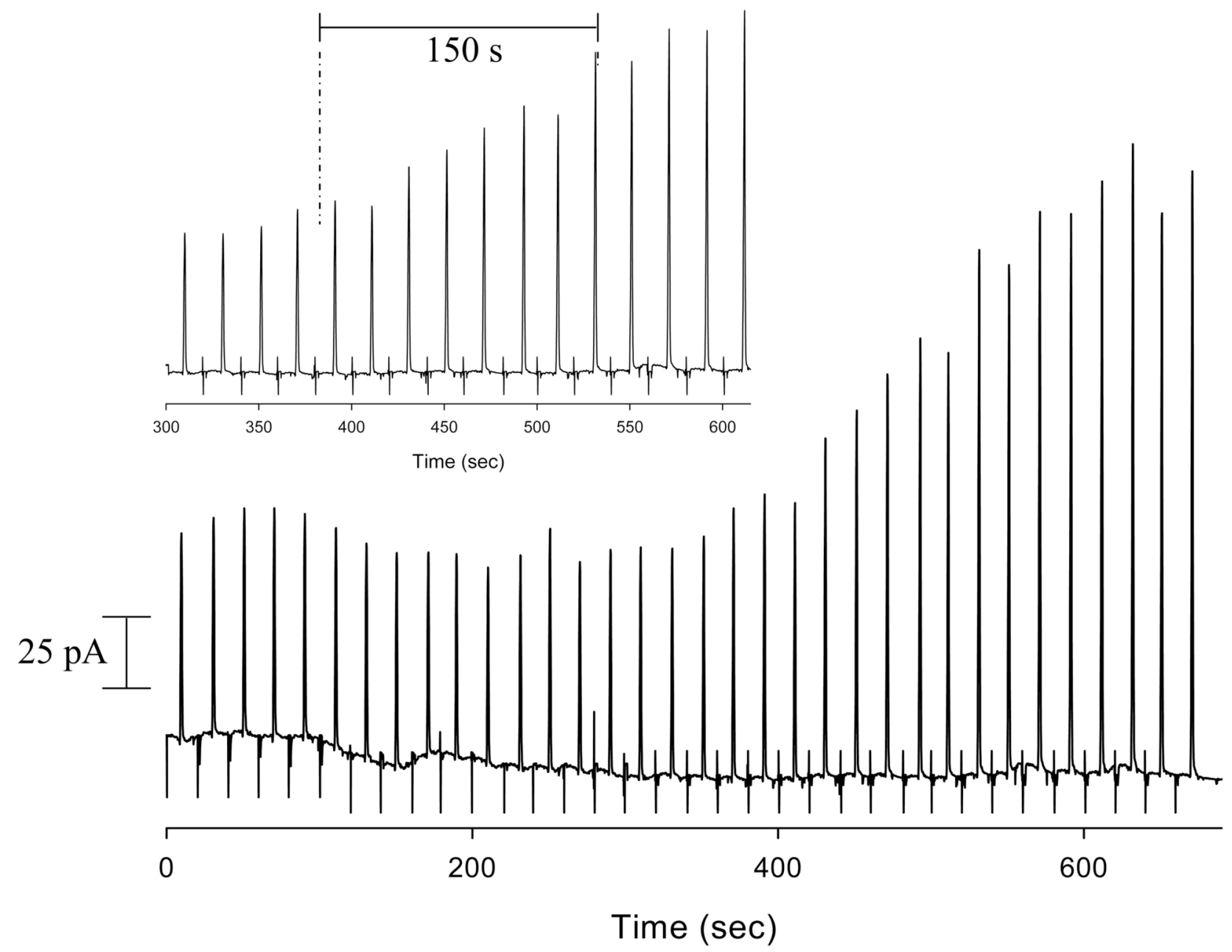

Figure 5.

Concentration change: continuous injections and amperometric detection $(+0.9 \mathrm{~V})$ of DA as sampled through a $5 \mathrm{~mm}$ linear probe and directly injected into the electrophoresis channel using MD/ME/EC device. Dopamine concentration was changed at $t=0$ from 100 to 300 $\mu \mathrm{M}$. Perfusate flow rate of $0.3 \mu \mathrm{L} / \mathrm{min}$. Electrophoresis buffer $=25 \mathrm{mM}$ boric acid with $3 \mathrm{mM}$ SDS (pH 9.5). Injection sequence: $400 \mathrm{msec}$ valve actuation with $2 \mathrm{sec}$ high voltage shut-off repeated every $20 \mathrm{sec}$. The lag time for this experiment was $330 \mathrm{sec}$. Inset shows zoomed in region depicting the rise time of $150 \mathrm{~s}$. 

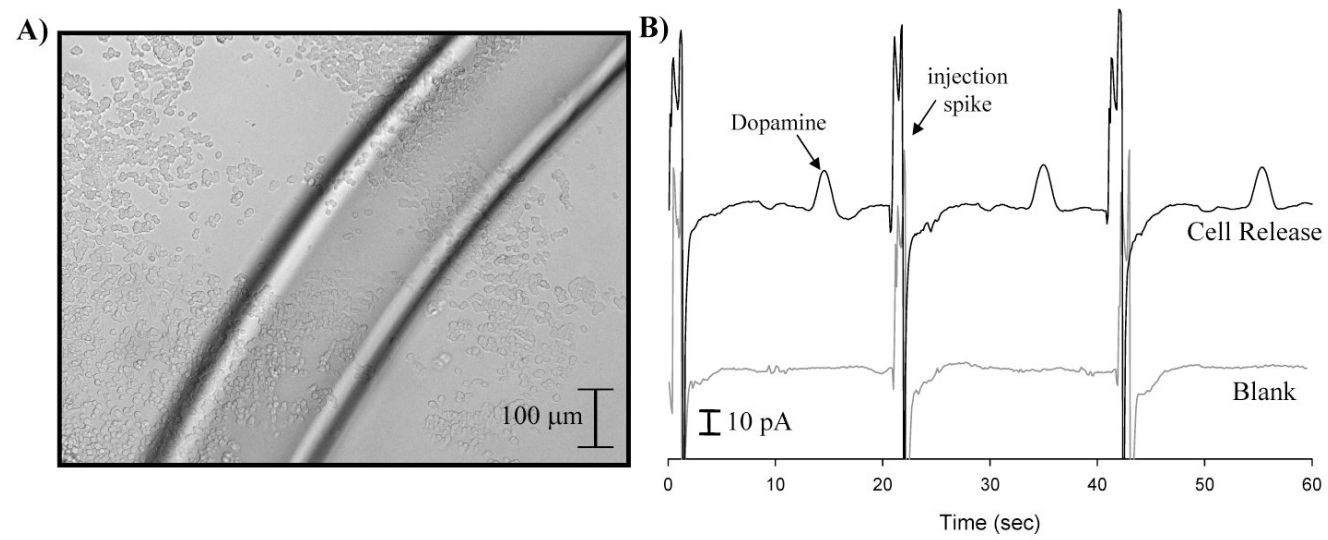

Figure 6.

A) Micrograph of PC 12 cells in $35 \mathrm{~mm}$ Petri dish with a $5 \mathrm{~mm}$ linear probe membrane lying over the cells and sampling the stimulated cellular release. B) Electropherogram of cell release while in buffered solution before stimulation (blank) and after the addition of stimulant solution as sampled, separated, and detected with MD/ME/EC system. Sampled at a perfusate flow rate of $0.3 \mu \mathrm{l} / \mathrm{min}$. Injection sequence: $800 \mathrm{msec}$ valve actuation with $2 \mathrm{sec}$ high voltage shut-off repeated every $20 \mathrm{sec}$. Electrophoresis buffer $=25 \mathrm{mM}$ boric acid with $3 \mathrm{mM} \mathrm{SDS}$ (pH 9.5). 